\title{
On Avoiding Vertexization of Robustness Problems: The Approximate Feasibility Concept
}

\author{
B. Ross Barmish \\ ECE Department, University of Wisconsin, Madison, WI 53706 \\ barmish@engr.wisc.edu \\ Pavel S. Shcherbakov \\ Institute of Control Science, Profsojuznaya, 65, Moscow 117806, Russia \\ sherba@ipu.rssi.ru
}

\begin{abstract}
For a large class of robustness problems with uncertain parameter vector $q$ confined to a box $Q$, there are many papers providing results along the following lines: The desired performance specification is robustly satisfied for all $q \in Q$ if and only if it is satisfied at each vertex $q^{i}$ of $Q$. Since the number of vertices of $Q$ explodes combinatorically with the dimension of $q$, the computation associated with the implementation of such results is often intractable. The main point of this paper is to introduce a new approach to such problems. To this end, the definition of approximate feasibility is introduced, and the theory which follows from this definition is vertex-free.
\end{abstract}

\section{Introduction}

In this paper, we consider robustness problems for systems described in terms of a design vector $x \in X \subseteq \mathbf{R}^{n}$ and a real uncertain parameter vector $q \in Q \subset \mathbf{R}^{\ell}$, where $Q$ is a box. For such systems, the objective is to select $x \in X$ such that a given continuous performance specification

$$
f(x, q) \leq 0
$$

is satisfied for all $q \in Q$. When such a design vector $x$ exists, the triple $(f, X, Q)$ is said to be robustly feasible. For the case when a design vector $x \in X$ exists leading to strict inequality, this triple is said to be strictly robustly feasible. Equivalently, there exists some $\sigma>0$ such that

$$
f(x, q) \leq-\sigma
$$

for all $q \in Q$. There are a large number of papers in the literature with robust feasibility formulations which fit into this framework; e.g., see [1]-[5]. Later in this section, we provide three examples illustrating how this formulation arises.

1.1 Vertexization: In many papers, it is shown that the robust satisfaction of $f(x, q) \leq 0$ is guaranteed if an only if $f\left(x, q^{i}\right) \leq 0$ for each of the vertices $q^{i}$ of the $\ell$-dimensional box $Q$. Henceforth, we use the word vertexization to describe a large number of such results in this literature. The takeoff point for this paper is the fact that as the dimension $\ell$ of $q$ increases, the number of vertices, $N=2^{\ell}$, undergoes a so-called combinatoric explosion. Consequently, the computational requirements associated with a vertexization result may be excessive. One well-known example illustrating this situation involves the failure of Matlab's LMI toolbox which can result; i.e., for a linear matrix inequality (LMI) involving even a modest number of uncertain parameters, the vertexization which is typically used can lead to a computational burden which cannot be handled with the existing code; e.g., see [4] for further discussion of this issue.

1.2 Approximate Feasibility: The main objective of this paper is to describe a new approach to robust design problems which is aimed at overcoming the type of computational intractability problems described above. Central to this new approach is the notion of approximate feasibility. This new concept, introduced in [3] in the restricted context of an LMI, involves softening the robustness formulation so as to allow an arbitrarily small volume $\epsilon>0$ of performance violation in the space of uncertain parameters. After formalization of the theoretical framework, it is seen that the approximate feasibility problem lends itself to a vertex-free solution. This avoidance of the vertex combinatorics is a main focal point of this paper.

1.3 Overview of Paper: In Section 2, to further motivate the theory to follow, we provide three examples of well-known robustness problems which all have the vertexization property discussed above. In Section 3 , the formal definition of approximate feasibility is given and concepts are illustrated via simple example. Section 4 is devoted to the notion of "homogenization" and the main result is given in Section 5. For a large class of homogenizable robustness problems described by $(f, X, Q)$, it is shown that their approximate feasibility counterparts are solvable via minimization of an appropriately constructed convex function $\Phi$. This convex optimization problem is subsequently illustrated in Section 6 by re-visiting the motivating examples given in Section 2. Section 7 considers the conditions under which approximate feasibility is equivalent to robust feasibility. Finally, in Section 8, future research is discussed. 


\section{Three Motivating Examples}

2.1 Example (Vertexization of Robust Quadratic Stability): Consider the famous quadratic stability problem with uncertain parameter vector $q \in Q$, uncertain $n \times n$ state space matrix

$$
A(q)=A_{0}+\sum_{i=1}^{\ell} A_{i} q_{i}
$$

being the affine linear combination of fixed matrices as indicated above and symmetric candidate Lyapunov matrix $P=P(x)$ with entries $x_{i} \in \mathbf{R}$ viewed as the design variables. Then, the problem of robust quadratic stability is to select a design vector $x \in X=\mathbf{R}^{n}$ such that $P(x)>0$ and

$$
.^{T}(q) P(x)+P(x) A(q)<0
$$

for all $q \in Q$. Hence, with

$$
f(x, q)=\lambda_{\max }\left(A^{T}(q) P(x)+P(x) A(q)\right),
$$

it is well known (for example, see [1]) that this strict feasibility design problem for $x$ is reducible to the vertices $q^{i}$ of $Q$. That is, the satisfaction of the Lyapunov inequality above for all $q \in Q$ is equivalent to $A^{T}\left(q^{i}\right) P(x)+P(x) A\left(q^{i}\right)<0$ for $i=1,2, \ldots, N$. This result, and an analogous result for a more general linear matrix inequality, is the basis for numerical solution of the problem. That is, one considers a "large LMI" by stacking the individual vertex LMIs. However, since $N=2^{\ell}$, we see that the computational task can easily get out of hand. For example, with five states and ten uncertain parameters, the resulting LMI is of size greater than $5000 \times 5000$.

2.2 Example (Vertexization of Robust Least Squares): Many robustness problems reduce to least squares problems. Indeed, with uncertain parameter vector $q \in Q$, uncertain $m \times n$ matrix $A(q)$, uncertain $m \times 1$ vector $b(q)$ and prespecified error tolerance $\delta>0$, the robust least squares problem (for example, see [5]) is to find a design vector $x \in X=\mathbf{R}^{n}$ such that

$$
\|A(q) x-b(q)\|^{2} \leq \delta^{2}
$$

for all $q \in Q$. Letting

$$
f(x, q)=\|A(q) x-b(q)\|^{2}-\delta^{2}
$$

to make a connection with the notation in this paper, the key observation to make is that if $A(q)$ and $b(q)$ depend affine linearly on $q$, the left-hand side above is convex in $q$. This implies that this inequality is satisfied for all $q \in Q$ if and only if $\left\|A\left(q^{i}\right) x-b\left(q^{i}\right)\right\|^{2} \leq \delta^{2}$ at each vertex $q^{i}$ of $Q$. Analogous to the case of the quadratic stability above, a computational scheme based on this vertexization may be impractical to carry out.

2.3 Example (Vertexization of Uncertain Linear Inequalities): With $q \in Q, A(q)$ and $b(q)$ as defined above, many robustness problems can be reduced to finding a robustly feasible solution for the set of uncertain linear inequalities. More specifically, with constraint set $X$ being a polyhedron, the robust feasibility problem for linear inequalities is to find a design vector $x \in X$ such that

$$
A(q) x \leq b(q)
$$

for all $q \in Q$. Note that this problem is described in terms of the formulation in this paper by taking

$$
f(x, q)=\max _{i} \eta_{i}^{T}(A(q) x-b(q)),
$$

where $\eta_{i}$ denotes a unit vector in the $i$-th co-ordinate direction. Moreover, analogous to the robust least squares problem above, it is readily shown that if $A(q)$ and $b(q)$ depend affine linearly on $q$, the desired set of linear inequalities is satisfied for all $q \in Q$ if and only if $A\left(q^{i}\right) x \leq b\left(q^{i}\right)$ at each vertex $q^{i}$ of $Q$.

\section{Approximate Feasibility}

As indicated in the introduction, our approach to computational intractability problems involves softening the robustness formulation so as to allow an arbitrarily small volume $\epsilon>0$ of performance violation in the space of uncertain parameters. We now formalize this idea.

3.1 Approximate Feasibility: We say that $(f, X, Q)$ is approximately feasible if the following condition holds: Given any $\epsilon>0$, there exists some $x^{\epsilon} \in X$ such that

$$
\operatorname{Vol}\left(\left\{q \in Q: f\left(x^{\epsilon}, q\right)>0\right\}\right)<\epsilon .
$$

We call $x^{\epsilon}$ an $\epsilon$-approximate solver. As indicated above, instead of guaranteeing satisfaction of $f(x, q) \leq 0$ for all $q \in Q$, we seek solution vectors $x$ with associated violation set

$$
Q_{b a d}(x) \doteq\{q \in Q: f(x, q)>0\}
$$

having volume less than any arbitrarily small prespecified level $\epsilon>0$. Analogous to the case of robustness, we say that $(f, X, Q)$ is strictly approximately feasible if there exists some $\sigma>0$ such that the following condition holds: Given any $\epsilon>0$, there exists some $x^{\epsilon} \in X$ such that

$$
\operatorname{Vol}\left(\left\{q \in Q: f\left(x^{\epsilon}, q\right)>-\sigma\right\}\right)<\epsilon .
$$

One of the main objectives of this paper is the problem of generating $\epsilon$-approximate solvers.

3.2 Approximate Feasibility Versus Robust Feasibility: Although robust feasibility trivially implies approximate feasibility (if $x^{\text {feas }}$ is feasible, take $x^{\epsilon}=$ $x^{\text {feas }}$ for all $\epsilon>0$ ), there are simple examples to show that the converse is false. To illustrate, for the LMItype scalar problem of [3] described by

$$
f(x, q)=1-x q^{2},
$$

$X=\mathbf{R}$ and $|q| \leq r$ defining $Q$, a straightforward calculation leads to

$$
\operatorname{Vol}\left(Q_{b a d}(x)\right)=2 r \quad \text { for } x \leq 0
$$


and

$$
\operatorname{Vol}\left(Q_{b a d}(x)\right)=2 \min \left\{r, \frac{1}{\sqrt{x}}\right\} \quad \text { for } x>0 .
$$

Hence, $(f, X, Q)$ is approximately feasible but not robustly feasible; see Section 7 for a result on the relationship between robust and approximate feasibility.

3.3 Motivation of Theory to Follow: To motivate the more formal technical exposition to follow in Sections 4 and 5 , we first illustrate our theory on a simple scalar example. To this end, we now compare the common sense solution method with the formal solution given in this paper. With the ideas associated with this example in mind, it is felt that the generalization to follow is more transparent.

Indeed, we consider a simple scalar LMI for strict robust feasibility described by $f(x, q)=1+x g(q)$ with $g(q)$ being a continuous, possibly nonlinear, function and $Q$ defined by $|q| \leq r$. By inspection, with $X=\mathbf{R},(f, X, Q)$ is strictly robustly feasible if and only if $g(q)$ has one sign. In such a case, letting $g^{*} \doteq \min _{|q| \leq r}|g(q)|>0$, it follows that feasible solutions $x=x^{f e a s}$ are completely characterized by $x^{f e a s}=-\rho \operatorname{sgn} g(0)$ with $\rho>1 / g^{*}$.

By way of contrast, we now solve this same problem by considering the specialization of theory to follow to the simple scalar problem at hand. To this end, we consider the function

$$
\Phi(x) \doteq \int_{-r}^{r} e^{1+x g(q)} d q
$$

in lieu of $f(x, q)=1+x g(q)$. To motivate the formalism to follow, we make three key observations: First, $\Phi(x)$ is a convex function of $x$. Second, in view of the simply derived inequality

$$
\operatorname{Vol}\left(Q_{b a d}(x)\right) \leq \int_{-r}^{r} e^{1+x g(q)} d q=\Phi(x),
$$

it follows that $(f, X, Q)$ is approximately feasible if $\Phi(x)$ can be made arbitrarily small by choice of $x$. That is,

$$
\Phi^{*} \doteq \inf _{x} \Phi(x)=0
$$

implies approximate feasibility. Third, if $\Phi^{*}=0$, we can use any iteration sequence $x_{k}$ leading to the minimum value of the convex function $\Phi(x)$ to obtain an $\epsilon$-approximate solver. That is, given any $\epsilon>0$, by picking $k$ suitably large so as to guarantee

$$
\Phi\left(x_{k}\right)=\int_{-r}^{r} e^{1+x_{k} g(q)} d q<\epsilon,
$$

and by taking $x^{\epsilon}=x_{k}$, we have obtained an $\epsilon$-approximate solver.

To complete this analysis, we illustrate the ideas above using the specific function $g(q)=1+q$ with $r<1$. Then, via a straightforward integration, we obtain $\Phi(x)=$ $\left(2 e^{1+x} \sinh r x\right) / x$ as the function to be minimized via our method. To see how the minimization of this function leads to an $\epsilon$-approximate solver, we first observe that $x$ achieves strict robust feasibility if and only if $x<-1 /(1-r)$. For such $x, \operatorname{Vol}\left(Q_{b a d}(x)\right)=0 ;$ similarly, for $x \geq-1 /(1+r)$ we have $\operatorname{Vol}\left(Q_{b a d}(x)\right)=2 r$. Finally, for the nontrivial case when $-1 /(1-r) \leq x<$ $-1 /(1+r)$, a simple calculation leads to $\operatorname{Vol}\left(Q_{\text {bad }}(x)\right) \leq$ $r-1-1 / x \leq \Phi(x)$.

It is apparent that a convex minimization of $\Phi(x)$ leads to an $\epsilon$-approximatesolver for any prescribed $\epsilon>0$. To illustrate, for a given $\epsilon>0$, suppose $x_{k} \rightarrow-\infty$ is a sequence obtained via minimization of $\Phi(x)$. Then, it follows that an $\epsilon$-approximate solver has been found once $x_{k}$ decreases to the point that

$$
\frac{2 e^{1+x_{k}} \sinh r x_{k}}{x_{k}}<\epsilon .
$$

For example, if $r=0.75$ and $\epsilon=0.01 r$, we guarantee satisfaction of $\operatorname{Vol}\left(Q_{b a d}\left(x_{k}\right)\right)<\epsilon$ once $x_{k}<-13.26$.

\section{Approximate Feasibility Indicators and Homogenization}

Motivated by the observations in the previous section, we first introduce the class of test functions which play the key role in establishing approximate feasibility of robustness problems.

4.1 Approximate Feasibility Indicator (AFI): A continuous function $\phi: \mathbf{R} \rightarrow \mathbf{R}$ is said to be an $a p$ proximate feasibility indicator if it has the following properties:

$$
\begin{aligned}
& \text { 1. } \phi(\zeta) \geq 0 \text { for all } \zeta \in \mathbf{R} \\
& \text { 2. } \phi(\zeta)<1 \text { if and only if } \zeta<0 \\
& \text { 3. } \phi(\zeta) \rightarrow 0 \text { as } \zeta \rightarrow-\infty
\end{aligned}
$$

4.2 Remark: Note that the definition above does not depend on $(f, X, Q)$. However, as explained in Section 5.2, there are a number of reasons associated with numerical computation why it is advantageous to tailor the choice of approximate feasibility indicator $\phi(\zeta)$ to the specification $f(x, q)$.

4.3 Types of AFIs: The first type of AFI was already introduced in Section 3.3; it has the form $\phi(\zeta)=e^{\zeta}$. Indeed, Conditions 1-3 above hold, and this function is an AFI. Clearly, such modifications as $\phi(\zeta)=e^{\alpha \zeta}$ with $\alpha>0$ also satisfy the definition above and can be used as AFIs. Various piecewise linear functions can also be taken as AFI. For instance, the second type of AFI is described by

$$
\phi(\zeta)=\left\{\begin{array}{cc}
0 & \text { for } \zeta \leq-\beta \\
1+\zeta / \beta & \text { for } \zeta>-\beta
\end{array}\right.
$$

where $\beta>0$ is an adjustable parameter. 
Before stating the main result, we describe the class of homogenizable performance specification functions $f(x, q)$ to which the theory applies.

4.4 Homogenization: The function $f(x, q)$ is said to be homogenizable in $x$ if there exists a continuous function $f^{+}:(0, \infty) \times \mathbf{R}^{n} \times \mathbf{R}^{\ell} \rightarrow \mathbf{R}$ and a positive integer $k$ such that

$$
f^{+}\left(\gamma x_{0}, \gamma x, q\right)=\gamma^{k} f^{+}\left(x_{0}, x, q\right)
$$

and

$$
f^{+}\left(x_{0}, x, q\right)<0 \text { if and only if } f\left(\frac{x}{x_{0}}, q\right)<0
$$

for all $\gamma>0, x_{0}>0, x \in \mathbf{R}^{n}$ and $q \in Q$. The pair $\left(x_{0}, x\right)$ is called the extended design vector.

4.5 Example (LMI): To illustrate the homogenization concept, we consider the linear matrix inequality

$$
F_{0}(q)+\sum_{i=1}^{n} x_{i} F_{i}(q)<0
$$

where $F_{i}(q), i=0, \ldots, n$, are known continuous symmetric matrix functions of $q \in Q$. To assure negativedefiniteness above, we take

$$
f(x, q) \doteq \lambda_{\max }\left(F_{0}(q)+\sum_{i=1}^{n} x_{i} F_{i}(q)\right) .
$$

For the homogenization of $f(x, q)$, we take $k=1$ and

$$
f^{+}\left(x_{0}, x, q\right)=\lambda_{\max }\left(x_{0} F_{0}(q)+\sum_{i=1}^{n} x_{i} F_{i}(q)\right) .
$$

Note that for some special cases, no homogenization may be needed because $f(x, q)$ may already be homogeneous. For example, the quadratic stability problem (see Example 2.1), a special case of an LMI, corresponds to $F_{0}(q) \equiv 0$ above. In this case, we can take

$$
f^{+}\left(x_{0}, x, q\right)=f(x, q) \text {. }
$$

4.6 Example (Least Squares): In the least squares setup (see Example 2.2) with

$$
f(x, q) \doteq\|A(q) x-b(q)\|^{2}-\delta^{2},
$$

we obtain a homogenization with $k=2$ and

$$
f^{+}\left(x_{0}, x, q\right) \doteq\left\|A(q) x-b(q) x_{0}\right\|^{2}-\delta^{2} x_{0}^{2} .
$$

4.7 Example (Linear Inequalities): For the problem in Example 2.3 with the performance specification

$$
f(x, q)=\max _{i} \eta_{i}^{T}(A(q) x-b(q)),
$$

the natural homogenization

$$
f^{+}\left(x_{0}, x, q\right)=\max _{i} \eta_{i}^{T}\left(A(q) x-b(q) x_{0}\right)
$$

with $k=1$ can be used. In Section 6.2, we see that other homogenizations are possible and obtain a socalled extended AFI which proves to be convenient for computation.

\section{Main Result}

In the theorem to follow, we use AFI $\phi(\zeta)$ with argument

$$
\zeta=f^{+}\left(x_{0}, x, q\right)
$$

in the determination of approximate feasibility.

5.1 Theorem: Given the continuous homogenizable performance specification function $f(x, q), X=\mathbf{R}^{n}$ and an approximate feasibility indicator $\phi(\cdot)$, define

$$
\begin{aligned}
\Phi\left(x_{0}, x\right) & \doteq \int_{Q} \phi\left(f^{+}\left(x_{0}, x, q\right)\right) d q \\
\Phi^{*} & \doteq \inf _{x_{0}>0, x} \Phi\left(x_{0}, x\right) .
\end{aligned}
$$

and

Then the following holds:

(i) Strict robust feasibility of $(f, X, Q)$ implies $\Phi^{*}=0$; (ii) $\Phi^{*}=0$ implies approximate feasibility of $(f, X, Q)$; (iii) For any $x_{0}>0$ and $x \in \mathbf{R}^{n}$,

$$
\operatorname{Vol}\left(Q_{b a d}\left(\frac{x}{x_{0}}\right)\right) \leq \Phi\left(x_{0}, x\right) .
$$

Proof: To prove (i) it suffices to show that for any $\varepsilon>0$ there exist $x_{0}^{\varepsilon}>0$ and $x^{\varepsilon} \in \mathbf{R}^{n}$ such that $\Phi\left(x_{0}^{\varepsilon}, x^{\varepsilon}\right)<\varepsilon$. Indeed, by strict robust feasibility there exists some $x=$ $x^{f e a s} \in \mathbf{R}^{n}$ such that $f\left(x^{f e a s}, q\right)<0$ for all $q \in Q$. Letting $f^{+}\left(x_{0}, x, q\right)$ be the function obtained from $f(x, q)$ via the homogenizability assumption and in view of Condition 3 defining an approximate feasibility indicator $\phi$, it follows that with $\gamma$ suitably large, $x_{0}^{\varepsilon}=\gamma$ and $x^{\varepsilon}=\gamma x^{f e a s}$, we have

$$
\phi\left(f^{+}\left(x_{0}^{\varepsilon}, x^{\varepsilon}, q\right)\right)<\frac{\varepsilon}{\operatorname{Vol}(Q)}
$$

for all $q \in Q$. It now follows that

$$
\Phi\left(x_{0}^{\varepsilon}, x^{\varepsilon}\right)=\int_{Q} \phi\left(f^{+}\left(x_{0}^{\varepsilon}, x^{\varepsilon}, q\right)\right) d q<\int_{Q} \frac{\varepsilon}{\operatorname{Vol}(Q)} d q=\varepsilon .
$$

To prove (ii) and (iii), we fix arbitrary $x_{0}>0$ and $x \in \mathbf{R}^{n}$. Using the definition of $f^{+}\left(x_{0}, x, q\right)$ and basic facts defining the AFI, we obtain

$$
\begin{aligned}
\operatorname{Vol}\left(Q_{b a d}\left(\frac{x}{x_{0}}\right)\right) & =\operatorname{Vol}\left(\left\{q \in Q: f^{+}\left(x_{0}, x, q\right)>0\right\}\right) \\
& \leq \int_{Q} \phi\left(f^{+}\left(x_{0}, x, q\right)\right) d q=\Phi\left(x_{0}, x\right)
\end{aligned}
$$

The proof of the theorem is complete.

5.2 Remarks: The theorem above indicates that the approximate feasibility question can be recast as an optimization problem. In this regard, it is important to 
note that this optimization can often be accomplished via convex programming. Indeed, it can readily be shown that this is the case if $f^{+}\left(x_{0}, x, q\right)$ is convex in $\left(x_{0}, x\right)$, and $\phi(\zeta)$ is convex and monotonically increasing. Whereas the conditions in Theorem 5.1 for approximate feasibility do not depend on the choice of AFI $\phi$, the behavior of a numerical algorithm is a different matter. This is particularly true for many cases when the integral above is not computable in closed form and Monte Carlo integration is used. In this regard, there is considerable incentive to tailor the AFI $\phi$ in an appropriate manner to the specification $f$ in order to obtain a closed form for the integral, thereby avoiding Monte Carlo simulation entirely.

\section{Numerical Examples}

In this section, we consider two numerical examples. In the first example, the function $\Phi\left(x_{0}, x\right)$ is computed using the standard Monte Carlo technique for approximate calculation of integrals. Namely,

$$
\Phi\left(x_{0}, x\right) \approx \frac{1}{N} \sum_{j=1}^{N} e^{\lambda_{\max }\left(f^{+}\left(\boldsymbol{x}_{0}, x, q^{j}\right)\right)}
$$

where $q^{j}=\left(q_{1}^{j}, \ldots, q_{\ell}^{j}\right) \in Q, j=1, \ldots, N$, are samples for the uncertainty obtained via the uniform distribution. In the second example, we see that it is sometimes possible to work with a function which might appropriately be called an extended $A F I$. Although such a function does not formally satisfy the requirements for being an AFI, we prove that it can nevertheless be used to obtain results which are identical in nature to those given in Theorem 5.1 .

6.1 Quadratic Stability: This first example is taken from [3] where the special case of AFI theory was provided in the context of linear matrix inequalities. Indeed, we consider quadratic stability of the $n \times n$ interval matrix

$$
A=A_{0}+\Delta A, \quad\left|\Delta A_{i j}\right| \leq r \cdot s_{i j}, \quad i, j=1, \ldots, n,
$$

of [6] described by

$$
\begin{gathered}
A_{0}=\left(\begin{array}{rrr}
-2 & -2 & 0 \\
1 & 0 & 0^{-} \\
1 & 0 & -2
\end{array}\right), \\
S=\left(\begin{array}{rrr}
0.1651 & 0.9394 & 0.5691 \\
0.2451 & 0.4727 & 0.1457 \\
0.7004 & 0.4014 & 0.3141
\end{array}\right)
\end{gathered}
$$

and radius $r>0$. The goal is to determine if a positivedefinite matrix $P$ exists such that $A^{\mathrm{T}} P+P A<0$ for all admissible $A$.

To formulate using the notation in Sections 1-3, the optimization variable $x \in \mathbf{R}^{6}$ is composed of the six distinct entries of the symmetric positive-definite matrix $P$. Since this example involves nine uncertain parameters, the standard LMI technique requires solving an optimization problem described by an $M \times M$ matrix with $M=3 \times 2^{9}=1536$. Even such a moderate $3 \times 3$ problem pushes the limits of standard LMI solvers such as LMI Toolbox in MatLaB.

Now, with $r=1$, we demonstrate use of the method prescribed by Theorem 5.1; note that $\operatorname{Vol}(Q)=512$ in this case. We carried the convex minimization of $\Phi(x)$ with an exponential AFI $\phi(\zeta)=e^{\zeta}$ and using $N=400$ samples for each integration. We obtain $\Phi^{*} \approx 11.6107$ and conclude that there is no common stabilizing $P>0$ for the interval family (LMI is infeasible). This minimum value $\Phi^{*}$ was achieved with the positive-definite matrix

$$
P_{\varepsilon}=\left(\begin{array}{rrr}
19.5989 & 16.1542 & -4.6553 \\
16.1542 & 30.4427 & -2.1223 \\
-4.6553 & -2.1223 & 9.4100
\end{array}\right)
$$

In order to validate this result, we carried a large-scale Monte Carlo test with $N=100,000$ samples and obtained $\operatorname{Vol}\left(Q_{b a d}\left(P_{\varepsilon}\right)\right) \approx 1.1315$. As predicted by our theory, this quantity is less than $\Phi^{*}$.

In the second part of this experiment, we took $r=0.5$; this time, optimization resulted in $\Phi^{*} \approx 0$ and

$$
P_{0}=\left(\begin{array}{rrr}
2419.6 & 1228.7 & 19.3 \\
1228.7 & 5572.3 & -686.7 \\
19.3 & -686.7 & 1403.9
\end{array}\right) \text {. }
$$

In accordance with Theorem 3.1, we conclude that this LMI is approximately feasible. It also turns out that the matrix $P_{0}$ above establishes robust feasibility; this can be readily verified by checking the inequality $A^{\mathrm{T}} P_{0}+$ $P_{0} A<0$ at the extreme points of $Q$.

6.2 Extended AFI For Robust Linear Inequalities: We consider

$$
A(q) x \leq b(q)
$$

with $x \in \mathbf{R}^{n}$ being the design vector, $A(q)$ being an $m \times n$ affine linear matrix function and $b(q)$ being an $m \times 1$ affine linear vector function of the uncertainty $q \in \mathbf{R}^{\ell}$, $\left|q_{i}\right| \leq r_{i}$, i.e.,

$$
A(q)=A_{0}+\sum_{i=1}^{\ell} A_{i} q_{i} ; \quad b(q)=b_{0}+\sum_{i=1}^{\ell} b_{i} q_{i}
$$

with each $A_{i}$ being a fixed $m \times n$ matrix and each $b_{i}$ being a fixed $m \times 1$ vector.

6.2.1 The Function $\Phi:$ As discussed at the beginning of this section, we carry out the calculations for this example using a so-called extended AFI which is tailored to the structure at hand. To this end, we construct a function $\Phi\left(x_{0}, x\right)$ which majorizes the volume of $Q_{b a d}\left(\frac{x}{x_{0}}\right)$ and has the properties required in Theorem 5.1. Specifically, letting $\eta_{i}$ denote a unit vector in the $i$-th co-ordinate direction, for $x$ as above and $x_{0}>0$, we introduce the function

$$
\varphi\left(x_{0}, x, q\right) \doteq \sum_{i=1}^{m} e^{\eta_{i}^{T}\left(A(q) x-b(q) x_{0}\right)}
$$


which plays the role of $\phi\left(f^{+}\left(x_{0}, x, q\right)\right)$. This allows for the computation of the corresponding integral

$$
\Phi\left(x_{0}, x\right)=\int_{Q} \varphi\left(x_{0}, x, q\right) d q
$$

in closed form given by

$$
\Phi\left(x_{0}, x\right)=2^{\ell} \sum_{i=1}^{m} \prod_{j=1}^{\ell} \frac{\sinh \eta_{i}^{T} \beta_{j} r_{j}}{\eta_{i}^{T} \beta_{j}} e^{\frac{\eta_{i}^{T} \beta_{0}}{\ell}},
$$

where $\beta_{j} \doteq A_{j} x-b_{j} x_{0}, j=0,1, \ldots, \ell$.

We now consider $\Phi$ above in the context of the assertions of Theorem 5.1. We first claim that strict robust feasibility implies that $\Phi$ can be driven to zero. To this end, let $x^{\text {feas }}$ be a robust solution satisfying

$$
A(q) x^{f e a s}<b(q)
$$

for all $q \in Q$. In view of the fact that

$$
\inf _{x_{0}>0, x} \Phi\left(x_{0}, x\right) \leq \inf _{\rho>0} \Phi\left(\rho, \rho x^{f e a s}\right),
$$

it suffices to show that $\Phi\left(\rho, \rho x^{\text {feas }}\right)$ tends to zero with $\rho$ tending to $+\infty$. Indeed, since each linear inequality is satisfied separately by $x^{\text {feas }}$, we have

$$
\eta_{i}^{T}\left(A(q) x^{\text {feas }}-b(q)\right)<0
$$

for all $q \in Q$ and $i=1,2, \ldots, m$. From this, it is easily shown that

$$
\lim _{\rho \rightarrow+\infty} \Phi\left(\rho, \rho x^{f e a s}\right)=0 .
$$

This completes the proof of the claim.

We now assume that the infimum of $\Phi$ is zero and claim that with

$$
Q_{b a d}(x) \doteq\left\{q \in Q: \eta_{i}^{T}(A(q) x-b(q)) \geq 0 \text { for some } i\right\},
$$

the inequality

$$
\operatorname{Vol}\left(Q_{b a d}\left(\frac{x}{x_{0}}\right)\right) \leq \Phi\left(x_{0}, x\right)
$$

holds for all pairs $\left(x_{0}, x\right)$ with $x_{0}>0$. Indeed, defining the sets

$$
Q_{b a d, i}^{+}\left(x_{0}, x\right) \doteq\left\{q \in Q: \eta_{i}^{T}\left(A(q) x-b(q) x_{0}\right) \geq 0\right\}
$$

for $i=1,2, \ldots, m$, and

$Q_{b a d}^{+}\left(x_{0}, x\right) \doteq\left\{q \in Q: \eta_{i}^{T}\left(A(q) x-b(q) x_{0}\right) \geq 0\right.$ for some $\left.i\right\}$, we obtain the chain of inequalities

$$
\begin{aligned}
\operatorname{Vol}\left(Q_{b a d}\left(\frac{x}{x_{0}}\right)\right) & \leq \sum_{i=1}^{m} \operatorname{Vol}\left(Q_{b a d, i}^{+}\left(x_{0}, x\right)\right) \\
& \leq \sum_{i=1}^{m} \int_{Q} e^{\eta_{i}^{T}\left(A(q) x-b(q) x_{0}\right)} d q=\Phi\left(x_{0}, x\right) .
\end{aligned}
$$

\section{From Approximate to Robust Feasibility}

In this section, we address the issue of equivalence between approximate feasibility and robust feasibility. Although the admissible set $X$ for the design vector is assumed compact in the theorem below, it is noted that the results to follow can be extended to problems for which the triple is $(f, X, Q)$ is compactifiable. In such cases, there exists a compact set $X_{*} \subset \mathbf{R}^{n}$ such that approximate feasibility of $\left(f, X_{*}, Q\right)$ is equivalent to approximate feasibility of $(f, X, Q)$. Such an extension comes into play when $f(x, q)$ is homogeneous in $x$. The theorem below is readily established.

7.3 Theorem: Assume $X$ is compact. Then strict robust feasibility of $(f, X, Q)$ and strict approximate feasibility of $(f, X, Q)$ are equivalent.

\section{Future Research}

In Section 6, the benefits of working with an extended $A F I$ were demonstrated. It is felt that further research along these lines is needed. A second area for future research involves extension of the theory to the nonhomogenizable case. Finally, as a third area of research, we point to the numerical issues with the Monte Carlo integration in view of convex minimization which need to be performed.

\section{Acknowledgements}

Funding for this research was provided by the National Science Foundation under Grants Numbers ECS-9811051 and ECS-9418709.

\section{References}

[1] Boyd, S. L., L. El Ghaoui, E. Feron, and V. Balakrishnan (1994). Linear Matrix Inequalities in Systems and Control Theory, SIAM Studies in Applied Mathematics, vol. 15, SIAM, Philadelphia.

[2] Blondel, V. D. and J. N. Tsitsiklis (1999). "A Survey on Computational Complexity Results in Systems and Control," to appear.

[3] Barmish, B. R. and P. S. Shcherbakov (1999). "Linear Matrix Inequalities with Uncertain Parameters: The Approximate Feasibility Concept," Proc. Conf. Inf. Sci. Syst., Baltimore.

[4] Apkarian, P. R. and H. D. Tuan (1999). "Relaxations of Parameterized LMIs With Control Applications," Int. J. Robust Nonlin. Contr., 9, pp. 59-84.

[5] El Ghaoui, L. and H. Lebret (1997). "Robust SoIutions to Least-Squares Problems with Uncertain Data," SIAM J. Matrix Anal. Appl., 18, pp. 10351064.

[6] Lagoa, C. M., P. S. Shcherbakov and B. R. Barmish (1998). "Probabilistic Enhancement of Classical Robustness Margins: The Unirectangularity Concept," Syst. Contr. Lett., 35, pp. 31-43. 